\title{
OTIONOMICS
}

Revista de los Estudios de Economía y Empresa

Dossier «Redes sociales, economía y empresa»

CAMBIO TECNOLÓGICO Y NUEVAS DINÁMICAS DE COMUNICACIÓN

\section{La nueva era de los mercados financieros y su globalización}

\section{Hisabet Ruiz Dotras}

Profesora de los Estudios de Economía y Empresa (UOC)

RESUMEN La globalización de los mercados financieros ha tenido lugar a partir de una serie de factores o circunstancias que han permitido la integración de los mercados financieros. Uno de ellos es la desregulación financiera y la libertad de movimientos de capitales. Pero uno de los factores más relevantes en este cambio ha sido el avance tecnológico relativo a la conexión entre mercados, facilitando y unificando sistemas de liquidación y compensación de operaciones, agilizando la negociación con servicios integrados de varios mercados, y mejorando el binomio riesgo-rendimiento para los inversores.

Como resultado de este gran cambio en el entorno económico y en el sistema financiero y su dinámica de comunicación, ha sido inevitable la evolución y aparición de nuevos productos financieros caracterizados por la innovación financiera. La finalidad de estos nuevos productos no es más que cubrirse ante el inevitable incremento de la volatilidad y el mayor riesgo en el conjunto de operaciones financieras.

El aspecto negativo de la globalización y la elevada vinculación o interconexión entre mercados es el efecto contagio de las crisis financieras. El ejemplo más reciente es la crisis de las hipotecas sub-prime, que se inició en el 2007 en Estados Unidos y seguidamente desencadenó una reducción de liquidez en el mercado de crédito global, impactando en las economías de diferentes países y poniendo de manifiesto la debilidad del sistema financiero mundial y también su marco regulador.

PALABRAS CLAVE globalización; mercados financieros; innovación financiera; tecnologías de la información y comunicación; efecto contagio

\section{The new age of financial markets and their globalisation}

ABSTRACT The globalisation of financial markets has been based on several factors that have enabled their integration. Two of them are financial deregulation and free movement of capital. Especially relevant to this change has been technological progress regarding the connection between markets. This has encouraged and standardised systems for settling and compensating transactions, speeded up trading with integrated services from different markets, and improved the riskbenefit ratio for investors. 
As a result of such big change in the economic environment as well as the financial system and its communication patterns, new innovative financial products have been developed. Their aim is to provide protection from growing volatility and increased risk in all financial transactions.

The negative side of globalisation and the high interconnection between markets is the contagion effect of financial crises. The most recent example is the sub-prime crisis that sparked off in the United States in 2007. This caused a drop in liquidity on the global credit market, which in turn affected the economy of several countries, and showed the weakness of the global financial system and its regulatory framework.

KEYWORDS globalisation; financial markets; financial innovation; ICT; contagion effect

\section{La transformación de los mercados financieros en los últimos años}

A lo largo de los últimos años, los mercados financieros han sufrido una profunda transformación en todos los sentidos. Estos cambios se deben a una combinación de distintos factores relacionados entre ellos. Podemos clasificar estos componentes como factores del entorno, de la oferta y de la demanda de los mercados financieros.

Cuando hablamos de los factores del entorno, es obligado hacer referencia a una serie de elementos como son la desregulación y la desintermediación financiera, y la evolución de las tecnologías de la información y comunicación que han favorecido la interconexión entre los agentes. La combinación de estos elementos permite explicar una parte importante de los cambios que han sufrido los mercados financieros en los últimos años (Asli et al., 2009).

Hasta el final de los años setenta, el sistema financiero español, altamente bancarizado, estaba sometido a una fuerte regulación y control que limitaba la actuación en general. Dentro del marco de regulación bancaria había coeficientes de inversión obligatorios para financiar actividades de sectores prioritarios en condiciones más ventajosas. Los tipos de interés estaban fijados por ley, de forma que se creaba un diferencial mecánico de tipos de interés activos y pasivos. De este modo, no se aseguraban únicamente los beneficios de las entidades financieras, sino que, además, se dificultaba sustancialmente la competencia en los precios. La única competencia entre entidades financieras era la de volumen de negocio y, por este motivo, se configuró una estructura bancaria con una densa red de oficinas.

Por el contrario, el proceso de desintermediación de los últimos años, basado en la desaparición progresiva de intermediarios y su sustitución por mediadores, ha implicado un desarrollo creciente de los mercados financieros, -especialmente del de capitales-, y la consecuente pérdida de relevancia de los productos bancarios más tradicionales.

Así pues, se ha pasado de las rígidas regulaciones nacionales de los años setenta a un mercado internacional altamente desregulado y a un panorama completamente diferente.

El resultado de este nuevo marco, propiciado por la desregulación financiera, ha sido un notable incremento de la movilidad internacional de capitales, facilitando una asignación más eficiente de los recursos a nivel mundial, una mayor diversificación de riesgos y un estímulo al crecimiento de las economías de los mercados emergentes (Mishkin, 2009). Consecuentemente, la eliminación de fronteras entre los diferentes intermediarios y mercados ha comportados una oferta cada vez más importante de productos y servicios financieros.

Por el contrario, la mayor desregulación también ha provocado una mayor complejidad de los mercados y, como consecuencia, una mayor inestabilidad, interpretada como una gran volatilidad de los mercados financieros. La respuesta frente a esta inestabilidad ha sido un incremento en la demanda de productos destinados a protegerse ante las oscilaciones de los precios o, simplemente, enriquecerse mediante la especulación.

Otro factor importante en la transformación del sistema financiero es el cambio tecnológico. El principal coste de un intermediario financiero es la gestión de la información, la cual es necesaria para valorar los diferentes 
riesgos (especialmente el riesgo de impago) y para gestionar la compraventa de los títulos, el pago de las correspondientes obligaciones y la solución de los posibles conflictos e incumplimientos en los mandatos contractuales.

En las últimas décadas, el desarrollo de los mercados financieros ha permitido disponer de unos procesos de tratamiento y gestión de la información a un coste relativamente bajo. Los cambios en los sistemas de información han permitido la interconexión, el almacenamiento y la computación de más datos con mayor rapidez. Las redes de telecomunicación han extendido sus ramificaciones y han aumentado su capacidad. Paralelamente, los protocolos de intercambio de datos más fiables han hecho posible conectar sistemas electrónicos de manera más eficiente. Gracias al desarrollo de la informática y las redes de telecomunicación, ha sido posible ofrecer productos y procesos financieros a un coste sensiblemente más reducido (Issing, 2000).

El desarrollo de los mercados financieros ha permitido disponer de unos procesos de tratamiento y gestión de la información a un coste relativamente bajo. Gracias al desarrollo de la informática y las redes de telecomunicación, ha sido posible ofrecer productos y procesos financieros a un coste sensiblemente más reducido.

Paralelamente, las nuevas tecnologías han generado una integración mundial de los mercados que posibilita y fomenta la negociación ininterrumpida (las veinticuatro horas del día) de prácticamente la mayoría de activos financieros.

Las transacciones han pasado de ser realizadas por personas, a ser ejecutadas por ordenadores de alta potencia que usan un software sofisticado, en el cual los microsegundos pueden significar la diferencia entre ganar o perder millones de dinero. Son las "tecnologías inteligentes" las que se ocupan de realizar las decisiones de compra y venta dentro de la red, en base a parámetros preestablecidos proporcionados per gestores. Ante una ineficiencia en el mercado, las operaciones de arbitraje se pueden realizar de forma instantánea, garantizando así una mayor integración de los mercados financieros a través de la equiparación de sus precios.

Así pues, la tecnología es una de las razones por las que actualmente vivimos unos niveles de volatilidad, fluctuación e incertidumbre en los mercados financieros que superan con creces la volatilidad de la crisis anterior más similar.

\section{Ventajas y desventajas de la globalización financiera}

Según Bustelo (1999) por globalización financiera se entiende "la creciente dependencia financiera mutua entre los países del mundo ocasionada por el mayor volumen y variedad de las transacciones transfronterizas de flujos de capital".

La globalización, definida como una creciente integración e interconexión de varios mercados domésticos en un único mercado financiero internacional ha cambiado dramáticamente el entorno económico y ha modificado el sistema financiero. La liberalización del régimen financiero internacional y la proliferación de nuevas técnicas e instrumentos de tipo financiero, junto con el rápido desarrollo de las tecnologías de la información y comunicación, han jugado un papel crucial en el impulso de la globalización.

Este cambio de funcionamiento tiene sus ventajas y desventajas (Climent i Meneu, 1999). Un entorno financiero cada vez más integrado ha beneficiado a los países en el sentido de que aparecen nuevas oportunidades de inversión y financiación al exterior. Mientras que en el pasado la mayoría de bolsas de todo el mundo estaban limitadas por las fronteras nacionales y sus industrias específicas (por ejemplo, la Bolsa española era un mercado formado principalmente por bancos y eléctricas), hoy en día se puede observar una expansión internacional de los mercados financieros y una consolidación de las bolsas financieras mundiales cada vez más fuerte.

Una clara señal de ello es la sensibilidad de los índices bursátiles de diferentes centros financieros, cuando son arrastrados a la baja a resultas de cualquier información negativa que se dé en un mercado financiero nacional particular.

El proceso de integración comporta efectos muy positivos para las empresas y accionistas en todo el mundo. La globalización del mercado financiero permite a los inversores de cualquier país no restringirse a las posibilidades en los mercados del territorio nacional, sino que pueden colocar su capital en los diferentes mercados del mundo, aprovechando así alternativas de inversión a nivel internacional y diversificando el riesgo al máximo. 
Las empresas por su parte pueden captar recursos a costes menores de los que obtendrían en sus mercados locales y beneficiarse de grandes economías en escala. Es decir, como consecuencia de la interconexión de los mercados financieros se incrementa la eficiencia de todas las operaciones al reducir los costes y favorecer una mejor asignación de los recursos a escala mundial. El resultado es una mejora de las combinaciones riesgorendimiento para los inversores.

Otro elemento que caracteriza el proceso de globalización de los mercados financieros es la creciente institucionalización del sistema. Los inversores institucionales (fondos de pensiones, fondos de inversión, compañías de seguros, etc.) han pasado a dominar los mercados financieros en detrimento de los inversores individuales, pues los primeros son más propensos a transferir fondos a través de las fronteras nacionales, con objeto de mejorar la diversificación de sus carteras y/o beneficiarse de la infravaloración de algún activo financiero o la especulación. Como resultado de todo ello, es notable el peso predominante de las transacciones financieras en el mercado de divisas respecto de las transacciones comerciales.

Aun así, las principales desventajas o riesgos de esta globalización financiera se forman por el llamado efecto contagio. Según Forbes (2001) y Rigobon (2002), se define como contagio los cambios en los mecanismos de transmisión desencadenados durante un periodo de inestabilidad. Es decir, el incremento del grado de dependencia entre mercados después de un shock. La interconexión de los mercados y las instituciones financieras han traído una nueva forma de propagación de las crisis financieras.

Según el Fondo Monetario Internacional, el volumen y la rapidez de las transacciones han incrementado considerablemente la variabilidad de los precios de los activos. Esto aumenta el efecto de contagio entre mercados, provocado a veces por burbujas especulativas que mueven con fuerza los precios. De forma que un acontecimiento sistémico en un mercado se extiende rápidamente más allá de las fronteras de este mercado. Un hecho como la crisis en el mercado hipotecario norteamericano desencadenó rápidamente efectos negativos al resto de mercados mundiales; provocando una elevada y prolongada incertidumbre en la valoración del riesgo de insolvencia, que ha acabado repercutiendo en una brusca parada del ciclo del dinero.

\section{La innovación financiera}

La innovación financiera es un concepto que resulta impreciso de definir. A pesar de todo, se puede entender como el desarrollo - dentro de del sistema financiero- de nuevos productos, procesos y mercados que aumentan la eficiencia del sistema, o bien la hacen más completa. Se entiende por completa el hecho de dar respuesta a nuevas demandas de los diferentes agentes económicos (Tufano, 2003).

Hasta los años ochenta, las innovaciones financieras que se daban en los mercados iban orientadas a equilibrar o mejorar la relación del binomio precio-riesgo. Es decir, transferencia del riesgo de insolvencia, innovaciones para generar liquidez al sistema, o bien, instrumentos que ayudaran a incrementar los recursos propios o ajenos disponibles en las empresas.

Actualmente, la innovación introducida en los mercados financieros se ha desarrollado todavía más debido principalmente al incremento de la volatilidad en los tipos de interés, en el tipo de cambio y precios de los activos financieros. Otros factores que han influenciado en el desarrollo de la innovación financiera son los avances producidos en la informática y telecomunicaciones, la competencia creciente entre los intermediarios financieros, y la carencia de normativa legal y fiscal única que regule los mercados de manera global (Chou, 2004).

Dentro del proceso de innovación financiera se encuentra la construcción y elaboración de productos financieros, es decir, la ingeniería financiera. Se entiende como ingeniería financiera aquella parte de la gestión financiera que se basa en el diseño y la elaboración de productos financieros que tienen un objetivo específico (Engelen et al., 2010). No se trata de cualquier producto financiero sino de aquellos que se construyen a partir de los llamados productos derivados, principalmente futuros, opciones y permutas financieras (swaps).

En un clima de fuerte competencia entre operadores (bancos, agentes de cambio, brokers, intermediarios financieros, etc.) dentro de un sistema completamente integrado de los mercados financieros, es inevitable el incremento de la volatilidad y el mayor riesgo en el conjunto de operaciones financieras. Esta inestabilidad se 
produce porque muchas empresas e inversores demandan un tipo de activo para gestionar y minimizar estos riesgos.

Una de las innovaciones de los años ochenta, que cogió un gran impulso en los noventa y que en el 2000 se extendió por toda Europa fue la titulización de activos (Securitization) (Longstaff, 2010).

Se define como titulización de activos al agrupamiento de préstamos y la posterior reconversión y venta de otros títulos garantizados por estos préstamos iniciales. En particular, es una técnica financiera que consiste en transformar créditos en títulos financieros, emitidos y negociados en el mercado de capitales. De este modo, activos no líquidos se convierten en otros que pueden generar liquidez inmediata.

A pesar de todo, los últimos acontecimientos han sacado a la luz ciertas derivaciones e irregularidades en la utilización de la titulización de los créditos inmobiliarios. La carencia de regulación gubernamental del sector financiero, la laxa regulación y, en algunos casos, la regulación insuficiente, ha llevado a una dificultad generalizada de la valoración riesgo, que en algunos casos se ha convertido en una infravaloración de este (McBarnet, 2010).

\section{Conclusiones}

La expansión y transformación de los mercados financieros en las últimas décadas ha sido espectacular. Los diferentes procesos de desregulación financiera y la libertad de movimientos de capitales, junto con el rápido progreso técnico, principalmente de las telecomunicaciones y la tecnología de la información, han desencadenado una globalización de los mercados financieros y la evolución y aparición de nuevos productos financieros caracterizados por la innovación financiera.

El resultado de todos estos cambios ha generado un aumento sin precedentes en la dimensión y amplitud de los mercados de divisas, bonos y acciones. Además, los mercados derivados, inexistentes en la mayoría de países o con una importancia relativamente pequeña a principios de los ochenta, han aumentado sus volúmenes exponencialmente. Así pues, la introducción de las nuevas tecnologías en los mercados financieros ha conllevado a grandes e inesperados efectos en la estructura de los mercados financieros.

Un mayor grado de integración de los mercados financieros significa una mayor eficiencia en los mercados y una mejora de las combinaciones riesgo-rendimiento para los inversores. La globalización de los mercados facilita que los recursos se enfoquen buscando una relación óptima entre riesgo y rendimiento, propiciando mayor eficiencia en la asignación de recursos hacia las economías que, por su grado de desarrollo o su potencial, ofrezcan mejores perspectivas de rentabilidades.

A la vez, una mayor integración también supone una pérdida de autonomía política, el crecimiento de las transacciones financieras y la aparición de nuevos productos financieros, destinados a cobertura ante el aumento de volatilidad y en búsqueda de rentabilidades a corto plazo.

La principal desventaja o riesgo de la globalización financiera se encuentra en el llamado efecto contagio, que se manifiesta cuando surge una crisis en alguna zona económica y automáticamente sacude otros mercados financieros.

Mientras la crisis de la economía americana se desencadenaba a raíz de la burbuja inmobiliaria y las hipotecas sub-prime, el resto de economías desaceleraron su crecimiento y los precios de la vivienda se redujeron en muchos países. Como consecuencia, la mayoría de instituciones financieras estuvieron sometidas a grandes pérdidas y los mercados se vieron alterados por una elevada incertidumbre generalizada sobre la medida y la incidencia de las pérdidas. El resultado fue una parada brusca de la financiación, desencadenando ventas precipitadas, que contribuyeron a fuertes caídas en los precios de activos y nuevas pérdidas.

El balance final de una crisis que se inició en el 2007 en Estados Unidos y rápidamente se trasladó al resto de países es todavía desconocido, pero sí sabemos que el componente globalizador de los mercados financieros ha jugado un papel importante, impactando en variables económicas de la mayoría de países, como el crecimiento económico, los niveles de productividad, el nivel salarial y la tasa de paro, sin olvidar el nivel de deuda pública y los extremadamente bajos tipos de interés. 
Paralelamente al avance imparable de la tecnología, los mercados financieros se redimensionan en nombre de la innovación y la necesidad de crear dinero. De modo que si resulta que la causa fundamental de la crisis es realmente la tecnología, en lugar de las finanzas, la combinación de política monetaria y re-regulación parece ser que no será suficiente en el largo plazo. La tecnología es implacable y es evidente que nunca dejará de progresar, de forma que una política económica o monetaria convencional no podrá, por sí sola, abordar el nuevo panorama financiero.

\section{Referencias bibliográficas}

ASLI, T. B. et al. (2009). "Financial Institutions and Markets across Countries and over Time: Data and Analysis". Working paper. The World Bank.

BUSTELO, P. (1999). "Globalización Financiera y Riesgo Sistémico: Algunas Implicaciones de la Crisis Asiáticas". Reunión de Economía Mundial. Huelva.

CHOU, Y. K. (2004). "Technological revolutions and financial innovations". Working Paper. University of Melbourne.

CLIMENT, F.J.; MENEU, V. (1999). "La Globalización de los Mercados Financieros Internacionales". Working Paper. Universidad de Valencia.

ENGELEN, E.; ERTURK, I.; FROUD, J. (2010). "Reconceptualizing financial innovation: frame, conjuncture and bricolage". Economy and Society. Vol. 39, n. ${ }^{\circ}$ 1, págs. 33-63.

FORBES, K.; RIGOBON, R. (2001). "Measuring Contagion: Conceptual and Empirical Issues". International Financial Contagion, Kluwer Academic Publishers. Págs.480.

ISSING, O. (2000). "The globalisation of financial markets". [http://www.ecb.europa.eu/press/key/date/2000/ html/sp000912_2.en.html].

LONGSTAFF, F. A. (2010). "The subprime credit crisis and contagion in financial markets". Journal of Financial Economic. Vol. 97, n. ${ }^{\circ}$ 3, págs. 436-45.

MCBARNET, D (2010). "Financial Engineering or Legal Engineering?" Working Papers. University of Edinburgh, School of Law.

MISHKIN, F.S. (2009). "Globalization and financial development". Journal of Development Economics. Vol.89, n. ${ }^{\circ}$ 2, págs. 164-169.

RIGOBON, R. (2002). "International Financial: Theory and Evidence in Evolution”. The Research Foundation of The Association for Investment Management and Research.

TUFANO (2003). Handbook of the Economics of Finance. Elsevier North-Holland.

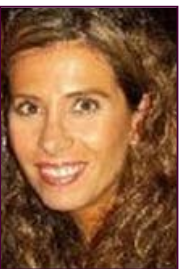

Flisabet Ruiz Dotras

eruizd@uoc.edu

Profesora de los Estudios de Economía y Empresa (UOC)

Av. Tibidabo, 39-43

Doctora en Ciencias Económicas y Empresariales por la Universidad de Barcelona. Licenciada en Administración y Dirección de Empresas por la Universidad Pompeu Fabra. Profesora agregada de los Estudios de Economía y Empresa de la Universitat Oberta de Catalunya (UOC), donde imparte asignaturas de finanzas y mercados financieros. Combina su actividad docente con la dirección académica del máster de Instrumentos y Mercados Financieros. Durante los últimos años ha compaginado la docencia e investigación con tareas de dirección académica de distintas titulaciones y la consultoría de valoración de activos financieros. 
Sus intereses de investigación están relacionados con la globalización de los mercados financieros, los tipos de interés, y las finanzas y el e-learning. Ha participado en varios proyectos nacionales, ha diseñado y creado cursos sobre mercados financieros y es autora de varios materiales y artículos sobre finanzas.

Es miembro del grupo de investigación Observatorio de la Nueva Economía (ONE) y del Management \& elearning (MEL).

Los textos publicados en esta revista están -si no se indica lo contrario- bajo una licencia Reconocimiento-Sin obras derivadas 3.0 España de Creative Commons. Puede copiarlos, distribuirlos y comunicarlos públicamente siempre que cite su autor y la revista y la institución que los publica (autoría, nombre de la revista, institución editora); no haga con ellos obras derivadas. La licencia completa se puede consultar en http://creativecommons.org/licenses/by-nd/3.0/es/deed.es.

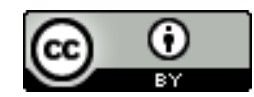

